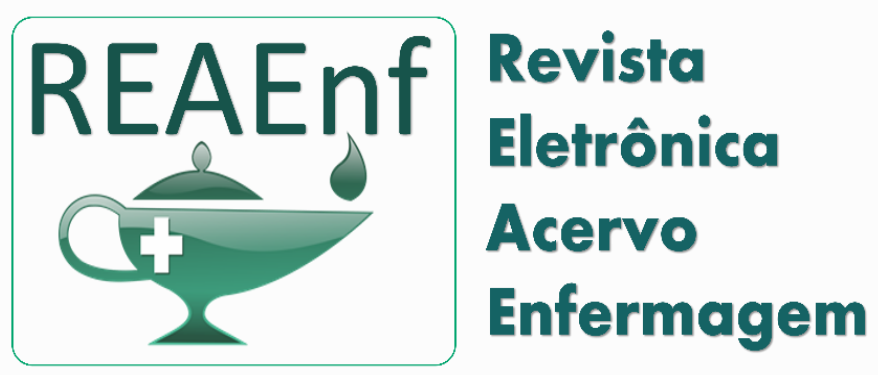

ARTIGO ORIGINAL

Recebido em: 10/2020

Aceito em: 11/2020

Publicado em: 12/2020

\title{
Caracterização das ocorrências do serviço de Atendimento Móvel de Urgência - SAMU
}

\author{
Characterization of occurrences of the Mobile Emergency Service - SAMU \\ Caracterización de incidencias del Servicio Móvil de Emergencia - SAMU \\ Regina Ribeiro de Castro ${ }^{1 *}$, Uemerson da Silva Faustino ${ }^{1}$, Daniel Matos Ribeiro ${ }^{1}$.
}

\begin{abstract}
Resumo: Esse artigo buscou caracterizar as ocorrências do Serviço de Atendimento Móvel de Urgência e Emergência por meio da pesquisa quantitativa, realizada com dados arquivados no Sistema de Informação do serviço. A amostragem selecionada correspondeu a quantidade de ocorrências atendidas nos meses de setembro à dezembro de 2019, e de janeiro à abril de 2020. A quantidade total de atendimentos no período de 2020 (6.158) foi bem próxima ao apresentado em 2019 (6.160). A média geral de atendimentos em 2019 e 2020 foi de 1.540 ocorrências. Em 2019 a USA apresentou a média de 258 atendimentos, da USB 1.197 e a motolância 45. A prevalência de atendimentos em 2020 realizados pela USB obteve a média 1.271, a USA 228 e a motolância 40. As ocorrências clínicas foram prevalentes em relação as outras naturezas. Porém, ressalta- se a incidência significativa dos serviços prestados às vítimas de acidente de trânsito, às vítimas de queda e aos pacientes internados que necessitaram de transferência inter-hospitalar.
\end{abstract}

Palavras-chave: Socorro de urgência, Emergências, Tele emergência.

\begin{abstract}
This article sought to characterize occurrences of the Mobile Emergency Care Service through quantitative research, carried out with data stored in the service's Information System. The correct sampling corresponded to the number of occurrences attended in the months of September to December 2019, and from January to April 2020. The total number of attendances in the period of $2020(6,158)$ was very close to that presented in $2019(6,160)$. The overall average of calls in 2019 and 2020 was 1.540 occurrences. In 2019 the USA has an average of 258 calls, from USB 1,197 and motolance 45 . The prevalence of calls in 2020 performed by USB obtained an average of 1,271, USA 228 and motolance 40 . Clinical occurrences were prevalent in relation to other types. However, the location of services provided to victims of traffic accidents, falls from falls and inpatients who required inter-hospital transfer is emphasized.
\end{abstract}

Keywords: Emergency relief, Emergencies, Tele emergency.

Resumen: Este artículo buscó caracterizar las ocurrencias del Servicio de Emergencias Móvil mediante una investigación cuantitativa, realizada con datos almacenados en el Sistema de Información del servicio. La muestra seleccionada correspondió al número de ocurrencias atendidas en los meses de septiembre a diciembre de 2019, y de enero a abril de 2020. El número total de asistencias en el período de 2020 (6.158) fue muy cercano al presentado en 2019 (6.160). El promedio general de llamadas en 2019 y 2020 fue de 1,540 ocurrencias. En 2019 USA presentó un promedio de 258 llamadas, de USB 1,197 y motolance 45. La prevalencia de llamadas en 2020 realizadas por USB obtuvo un promedio de 1,271, USA 228 y motolance 40. Las ocurrencias clínicas fueron prevalentes en relación a las demás. naturalezas. Sin embargo, se destaca la importante incidencia de servicios prestados a víctimas de accidentes de tráfico, víctimas de caídas y pacientes hospitalizados que precisaron traslado interhospitalario.

Palabras clave: Ayuda de emergencia, Emergencias, Tele emergencia.

${ }^{1}$ Centro Universitário UniEVANGÉLICA, Anápolis - GO. *E-mail: reginarc2008@hotmail.com 


\section{INTRODUÇÃO}

O perfil de atendimentos de urgência e emergência modifica- se com tempo, em decorrência da urbanização crescente, associado o aumento de causas violentas, tais como: agressões físicas, tentativas de homicídio e suicídio e outras causas externas acidentais e não acidentais, justificando a importância dos serviços de atendimento pré-hospitalar (APH) a vítimas, para prevenção de mortes evitáveis, complicações e sequelas incapacitantes (GOMES ATL, et al., 2017; MALTA DC, et al., 2017). Estudos que analisam as informações dessa modalidade da saúde, possibilitam o conhecimento sobre o trabalho realizado nos ambientes de $\mathrm{APH}$, além de apresentar características específicas do atendimento, favorecendo 0 planejamento e a implementações de ações nessa área da saúde.

O atendimento em saúde fora do hospital surgiu diante da necessidade de socorro às vítimas em ambientes e condições diversas. Com o transporte rápido e a adequada assistência aos soltados feridos em grandes guerras mundiais do passado, houve considerável redução da morbimortalidade, evidenciando a necessidade de implantação de um serviço de resgate de vítimas. A criação do APH no Brasil ocorreu nos modelos americano, contando com a atuação de técnicos em saúde; e do francês, sob regulação médica. Atualmente no Brasil, os serviços de urgências e emergências compõem-se em um Sistema de Rede de Atenção à Saúde, os quais fazem parte o serviço pré-hospitalar móvel, as unidades pré-hospitalares fixas, as unidades hospitalares de referência e as unidades pós-hospitalares de reabilitação (BRASIL, 2002).

O serviço de atendimento móvel de urgência (SAMU) está inserido na Política de Atenção as Urgências, Portarias 2048/2002 e 1863/2003. Ë uma modalidade de APH que atua junto as corporações militares de bombeiros e policiais e as agências privadas de resgate. O SAMU é realizado pelas equipes da Unidade de Suporte Avançado (USA), Unidade de Suporte Básico (USB), as Motolâncias, as Aeronaves, as Embarcações e as ambulâncias de transporte eletivo. A decisão sobre qual atendimento será destinado ao solicitante é de responsabilidade do médico regulador das urgências, podendo abranger a orientação para um cuidado específico, a procura de uma unidade de referência ou o envio da ambulância, dependendo da avaliação do quadro referido na ligação 192 (BRASIL, 2002). As solicitações pré-hospitalares e hospitalares de transportes passam pela regulação do SAMU, nas variedades de circunstâncias clínicas, cirúrgicas, psiquiátricas, obstétricas, ginecológicas, traumáticas e outras (BRASIL, 2002). Com interesse em aprofundar no conhecimento da demanda dos serviços realizados pelas equipes do SAMU, questiona-se qual o perfil das ocorrências atendidas e suas características?

Atualmente, os impactos causados pelo aumento da população e os avanços tecnológicos, principalmente nos transportes, têm favorecido a ocorrência dos traumas. Nesse contexto, o atendimento pré-hospitalar (APH) consiste em ações de saúde, realizadas ao doente ou a vítima, antes da chegada a unidade hospitalar. Tem como perfil agilidade e eficácia no atendimento as ocorrências de natureza clínica, cirúrgica, traumática, psiquiátrica, pediátrica, ginecológica, obstétrica e outras. Faz-se necessário que o APH seja realizado por profissional qualificado, no local do evento, durante o transporte até a chegada na instituição de saúde referenciada para o caso (GOMES ATL, et al, 2017).

Diante do aumento da população nas faixas etárias de envelhecimento, evidenciado nos censos demográficos do país, tem- se que os motivos de solicitação ou pedidos de socorro ao SAMU, ocorram em maior parte por causas clínicas de doenças crônicas não-transmissíveis, seguidas das causas traumáticas, frequentes nos acidentes de trânsito e ocorrências de violência (BRASIL, 2002; MALTA DC, et al., 2017). Assim, como forma de visibilizar a situação epidemiológica dos casos atendidos pelo SAMU em uma cidade de Goiás, buscou-se nessa pesquisa caracterizar as ocorrências do Serviço de Atendimento Móvel de Urgência e Emergência.

\section{MÉTODOS}

Esta é uma pesquisa quantitativa, realizada com dados do Serviço de Atendimento Móvel de Urgência (SAMU), em uma grande cidade do estado de Goiás. A amostragem selecionada corresponde a quantidade de ocorrências atendidas nos meses de setembro à dezembro de 2019 e de janeiro à abril de 2020, os quais correspondem ao número de atendimento realizado no ano e respectivos meses, o tipo de

REAEnf/EJNC | Vol. 7 | e5625 | DOI: https://doi.org/10.25248/REAenf.e5625.2020 Página 2 de 7 
atendimento oferecido, unidade de suporte avançado (USA) ou unidade de suporte básico (USB), o local de ocorrência, o motivo ou causa da solicitação, bem como o destino ou unidade de referência de transporte do paciente. Para discussão dos resultados foram selecionadas referências científicas que abordam o tema, publicados em Base de Dados da Biblioteca Virtual em Saúde, datados a partir de 2016.

A pesquisa tratada por meio da análise de dados quantitativos, possibilita a produção de conhecimento com base científica sólida e fortalecida, embasada por pelo estudo e reflexões de outros autores. Na saúde, os dados quantitativos potencializam a visão do cenário real, que por sua vez é dinâmico e relacional na população. As variáveis são apresentadas em tabelas, abordadas de forma analítica e descritiva. Esse tipo de pesquisa permite o conhecimento profundo da situação, podendo estabelecer fatores causais de um agravo e assim viabilizar planos e práticas em saúde adaptadas às características e necessidades da população (ESPERÓN JMT, 2017).

O local de pesquisa estabelecido foi a Base do SAMU na Região de Saúde. Esse corresponde ao serviço que compõe a rede de atenção às urgências e emergências, com toda a estrutura, recursos e dinâmica de trabalho estabelecidos pelas recomendações e orientações do Ministério da Saúde, descritas nas Portarias 1863/2003 e a 2048/2002 (BRASIL, 2002; BRASIL, 2003). A regulação do SAMU possui abrangência para 11 municípios, com o telefone de contato principal 192. Funciona 24 horas e conta com profissionais qualificados nas categorias de médicos, enfermeiros, técnicos de enfermagem, atendentes de telefonia, condutores socorristas e a equipe administrativa.

A coleta de dados foi realizada após as aprovações da Coordenação de Pesquisa na Secretaria Municipal de Saúde, da Direção do Curso de Enfermagem da UniEVANGÉLICA e do Comitê de Ética em Pesquisa Envolvendo Seres Humanos da UniEVANGÉLICA (CAAE número 24738819.0.0000.5076), obedecendo as determinações da Resolução 466/2012. Os dados foram fornecidos pela coordenação do SAMU em arquivos da planilha Microsoft Excel 2010, copiados para os documentos dos autores e assim categorizados e quantificados em valores absolutos e relativos, apresentados nas tabelas.

\section{RESULTADOS}

A quantidade total de atendimentos no período de 2020 (6.158) foi bem próxima ao apresentado em 2019 (6.160). A amostragem analisada nos períodos de 2019 e 2020 corresponderam as variáveis de maior frequência nos registros disponibilizados. Observou- se que no período analisado de 2019 houve maior demanda de chamada para o SAMU no mês de novembro e a menor procura de atendimento no mês de dezembro. A média geral de atendimentos foi de 1.540, da USA 258, da USB 1.197 e da motolância 45 (Tabela 1).

Tabela 1 - Distribuição dos atendimentos realizados pelas ambulâncias do SAMU, nos meses de setembro a dezembro de 2019.

\begin{tabular}{ccccccc}
\hline Meses / Ambulâncias & Set & Out & Nov & Dez & Total & $\%$ \\
\hline USA & 289 & 292 & 395 & 216 & 1192 & 19,3 \\
\hline USB & 1239 & 1255 & 1439 & 854 & 4787 & 77,7 \\
Motolância & 25 & 57 & 52 & 47 & 181 & 2,9 \\
\hline Total & 1553 & 1604 & 1886 & 1117 & 6160 & 100 \\
$\%$ & 25,2 & 26,1 & 30,6 & 18,1 & 100 & -
\end{tabular}

Legenda: USA: unidade de suporte avançado; USB: unidade de suporte básico.

Fonte: CASTRO RR, et al., 2020.

Nessa amostragem, as naturezas analisadas corresponderam a $83,2 \%$ do total das ocorrências realizadas pelo SAMU no período. As ocorrências clínicas foram prevalentes, porém ressalta- se a incidência significativa dos serviços prestados, em $2^{\circ}$ lugar às vítimas de acidente de trânsito, $3^{\circ}$ às vítimas de queda e $4^{\circ}$ aos pacientes internados que necessitaram de transferência inter-hospitalar. Outro dado importante, diz respeito as unidades de referência de transporte do paciente, de acordo com o quadro de saúde apresentado, em que a Unidade Pré-hospitalar Fixa recebeu a maioria dos pacientes, seguida do Hospital Municipal e Hospital Geral Filantrópico (Tabela 2). 
Tabela 2 - Distribuição dos atendimentos realizados pelo SAMU, conforme a natureza e os locais de referência, no ano de 2019.

\begin{tabular}{ccccccc}
\hline Meses & Set & Out & Nov & Dez & Total & $\%$ \\
\hline Natureza & & & & & & \\
\hline Clínica & 770 & 878 & 979 & 701 & 3328 & 64,9 \\
Acidente de trânsito & 192 & 184 & 205 & 206 & 787 & 15,3 \\
Queda & 102 & 90 & 136 & 212 & 540 & 10,5 \\
Transferência & 125 & 116 & 195 & 37 & 473 & 9,2 \\
\hline Total & 1189 & 1268 & 1515 & 1156 & 5128 & 100 \\
\hline \% do total geral do período: 6160 & 19,3 & 20,6 & 24,6 & 18,8 & 83,2 & - \\
\hline Destino & & & & & & \\
\hline Unidade Pré-hospitalar Fixa & 502 & 552 & 585 & 452 & 2091 & 49,7 \\
Hospital Municipal & 263 & 268 & 283 & 112 & 926 & 22 \\
Hospital Geral Filantrópico & 121 & 112 & 135 & 112 & 480 & 11,4 \\
Hospital de Urgências & 95 & 105 & 142 & 103 & 445 & 10,6 \\
Hospital Privado & 62 & 59 & 89 & 53 & 263 & 6,2 \\
\hline Total & 1043 & 1096 & 1234 & 832 & 4205 & 100 \\
\hline
\end{tabular}

Fonte: CASTRO RR, et al., 2020.

Em 2020, obteve-se a média geral de 1.540 atendimentos na amostragem analisada, com elevada prevalência de atendimentos realizados pela USB, com a média de 1.271, a USA 228 e a motolância 40,5 (Tabela 3).

Tabela 3 - Distribuição dos atendimentos realizados pelas ambulâncias do SAMU, nos meses de janeiro a abril de 2020.

\begin{tabular}{ccccccc}
\hline Meses/ Ambulâncias & Jan & Fev & Mar & Abr & Total & $\%$ \\
\hline USA $^{*}$ & 256 & 218 & 210 & 229 & 913 & 14,8 \\
USB $^{*}$ & 1259 & 1281 & 1321 & 1222 & 5083 & 82,5 \\
Motolância $_{\text {Total }}^{53}$ & 36 & 38 & 35 & 162 & 2,6 \\
\hline$\%$ & 1568 & 1535 & 1569 & 1486 & 6158 & 100 \\
\hline & 25,5 & 24,9 & 25,5 & 24,1 & 100 & - \\
\hline
\end{tabular}

Legenda: USA: unidade de suporte avançado; USB: unidade de suporte básico.

Fonte: CASTRO RR, et al., 2020.

Em 2020, nos períodos analisados houve a prevalência das ocorrências clínicas, seguindo- se as quedas e os acidentes de trânsito. A Unidade Pré-hospitalar Fixa liderou como unidade de referência para encaminhamentos do SAMU, a seguir as unidades de referência Hospital de Urgências e Hospital Municipal, todas unidades públicas de saúde (Tabela 4).

Tabela 4 - Distribuição dos atendimentos realizados pelo SAMU, conforme a prevalência de registros da natureza, dos locais de atendimento no ano de 2020.

\begin{tabular}{ccccccc}
\hline Meses & Jan & Fev & Mar & Abr & Total & $\%$ \\
\hline Natureza & & & & & & \\
\hline Clínica & 691 & 644 & 701 & 645 & 2681 & 51,7 \\
Queda & 230 & 230 & 307 & 200 & 967 & 18,6 \\
Acidente de trânsito & 232 & 236 & 304 & 183 & 955 & 18,4 \\
Transferência & 202 & 205 & 89 & 89 & 585 & 11,3 \\
\hline Total & 1355 & 1315 & 1401 & 1117 & 5188 & 100 \\
\hline Destino & & & & & & \\
\hline Unidade Pré-hospitalar Fixa & 602 & 601 & 538 & 402 & 2143 & 48,5 \\
Hospital de Urgências & 299 & 234 & 138 & 156 & 827 & 18,7 \\
Hospital Municipal & 142 & 92 & 203 & 284 & 721 & 16,3 \\
Hospital Geral Filantrópico & 51 & 83 & 114 & 119 & 367 & 8,3 \\
Total & 94 & 102 & 78 & 90 & 364 & 8,2 \\
\hline
\end{tabular}

Fonte: CASTRO RR, et al., 2020. 


\section{DISCUSSÃO}

A prevalência de ocorrências atendidas pela USB comprova a alta demanda de atendimentos de urgência, sem risco de morte imediata ou com risco presumido, sem a necessidade de abordagem da equipe da USA (BRASIL, 2002; BATTISTI GR, et al., 2019; O'DWYER G, et al., 2017). As equipes de atendimento nas ambulâncias são compostas de profissionais de saúde médicos, enfermeiros, técnicos em enfermagem e os condutores. O sistema de regulação das urgências controla o envio das equipes, 0 atendimento no local e o direcionamento do paciente para a unidade de saúde referência.

Essa organização e qualificação do APH visa atender o doente com agilidade e competência, dentro dos princípios da integralidade, equidade e universalidade do Sistema Único de Saúde (SUS) (GOMES ATL, et al., 2017). No SAMU, a presença da equipe da motolância merece destaque, mesmo diante da menor incidência em relação as demais ambulâncias. É um serviço que se caracteriza pela qualidade e agilidade no atendimento. Essa equipe é acionada quando há a necessidade de avaliação qualificada do doente para tomada de decisão; quando se percebe a gravidade do doente e se julga o início rápido da assistência até a chegada da USA; e, quando existe elevado tráfego no trânsito dificultando a passagem da ambulância. A otimização do tempo de resposta é uma das qualificações que justificam o trabalho da equipe da motolância no SAMU (Portaria GM/MS no 2.971/08), prezando sempre na disponibilização do serviço pela segurança dos pilotos profissionais (BRASIL, s/d). As emergências quando atendidas precocemente favorecem a melhor assistência e recuperação do paciente, levando a redução de sequelas incapacitantes e mortes evitáveis. As urgências e emergências podem ser definidas como eventos vitais para o paciente, com essencial abordagem do APH aos doentes (GOMES ATL, et al., 2017).

Em janeiro de 2020, obteve-se a prevalência de atendimento de todas as ambulâncias, podendo corresponder a maior demanda relacionada ao mês de férias. Entretanto, os quatro meses iniciais de 2020, apresentaram redução no número de ocorrências atendidas pelas ambulâncias em relação a 2019, possivelmente, relacionada ao uso racional do serviço, decorrente da melhor regulação da oferta e/ou do melhor entendimento da população para o chamado em situação de urgência e emergência. Essa percepção da importância do uso racional dos recursos na saúde, devido à crescente demanda do APH. Contudo, evidencia-se no mundo uso irregular desse serviço, em que $10 \%$ das solicitações são concluídas com envio de ambulância e realmente justificam o atendimento (O'DWYER G, et al, 2017).

As transferências demandaram elevada quantidade de atendimento do SAMU. É um tipo correspondente ao APH secundário, caracterizado pelo transporte inter-hospitalar. Essas dizem respeito ao serviço que garante a integralidade e resolutividade da condição de agravo do doente, uma vez que o transporte qualificado em ambulâncias do SAMU para uma unidade de saúde indicada a sua necessidade, complementa o tratamento (BRASIL, 2002).

Almeida PMV, et al. (2016), referem que aproximadamente $82 \%$ dos pacientes atendidos no SAMU são direcionados para unidades hospitalares. Percebe-se a importância do adequado planejamento de referência e contra referência dos pacientes, bem como, a efetiva comunicação entre os componentes da rede de assistência do SUS. Na amostragem analisada nessa, sobressaíram os atendimentos por motivos clínicos. Independente do tipo de ambulância USA ou USB, é comprovado a elevada demanda por causas clínicas, seguida por ocorrências traumáticas, causadas por acidentes de trânsito e quedas (ALMEIDA PMV, et al., 2016). Nos atendimentos de porta, a Unidade Pré-hospitalar Fixa no município é responsável por atender grande parte dos casos clínicos, o Hospital Municipal os casos cirúrgicos e ortopédicos, além de ofertar leitos de internação geral e retaguarda, e o Hospital Geral Filantrópico oferta atendimentos clínicos, cirúrgicos, pediátricos, ortopédicos e leitos para internação.

Os APHs surgiram no Brasil a partir de demandas relacionadas às urgências traumáticas. Essa preocupação se deu pelos dados epidemiológicos que apontaram significativo aumento no número de vítimas de causas externas, representadas pelos acidentes de trânsito e violências. Essa condição se assemelha aos países desenvolvidos, onde a morbimortalidade por causas externas é frequente (GOMES ATL, et al, 2017).

REAEnf/EJNC | Vol. 7 | e5625 | DOI: https://doi.org/10.25248/REAenf.e5625.2020 Página 5 de 7 
Em 2019, os acidentes de trânsito representaram a segunda maior causa de solicitação do SAMU, alcançando o terceiro lugar em 2020. Os traumas originados dos acidentes de trânsito acometem principalmente a população jovem e adulta, com maior prevalência dos indivíduos do sexo masculino, envolvendo motocicleta ou automóvel, atingindo com maior frequência os membros inferiores (SANTOS LFS, et al, 2016). Vemos que os acidentes de trânsito correspondem a uma grande preocupação para a qualidade de vida das pessoas, uma vez que atingem com maior frequência a população em pleno desenvolvimento profissional e desempenho produtivo. Além da elevada incidência dos traumas por acidentes de trânsito, tem -se a grande demanda de traumas decorrentes das quedas.

Nessa pesquisa, as quedas ocuparam o quarto lugar nos últimos meses de 2019 e passou para o segundo lugar em 2020. Situação semelhante é apresentada por Almeida PMV, et al. (2016). As quedas são eventos traumáticos muito frequentes em crianças (masculino) e idosos (feminino), possuindo como principal ambiente de ocorrência o domicílio. A presença pregressa de doença crônica (hipertensão arterial e diabetes) eleva a possibilidade de queda do idoso. A complicação maior é que quase a totalidade das vítimas de queda apresenta lesão física associada, de gravidade variável. A mortalidade desse agravo, possui maior incidência nas vítimas idosas, estando associada aos traumas de quadril e crânio encefálico alcançando índice 9\% de mortalidade no mundo. (RIBEIRO AP, et al., 2016; SANTOS LFS, et al., 2016; TIENSOLI SD, et al., 2019).

Os impactos causados pelo aumento da população e os avanços tecnológicos, principalmente nos transportes, tem favorecido a ocorrência dos traumas. Nesse contexto, o APH consiste em ações de saúde, realizadas ao doente ou vítima, antes da chegada a unidade hospitalar, a fim de proteger o paciente de complicações. Dessa forma, entende-se que o APH deve ser realizado por profissional qualificado, no local do evento e durante o transporte, até a chegada na instituição de saúde referenciada para o caso (GOMES ATL, et al., 2017). O SAMU corresponde a um serviço qualificado de APH, direcionado as demandas clínicas, traumáticas e outras, realizado por equipe multiprofissional no atendimento direto a população ou no direcionamento do doente para a unidade adequada ao seu tratamento. Com certeza é um serviço que veio para ficar e expandir em todo o território brasileiro, trazendo segurança na abordagem do doente.

\section{CONCLUSÃO}

$\mathrm{Na}$ quantificação dos atendimentos realizados pelas equipes do SAMU foi possível perceber o grande número de pessoas que usufruem desse serviço. Grande parte desse é desenvolvido pela equipe da UBS, composta pelo condutor socorrista e pelo técnico de enfermagem, na assistência ao paciente em condições de urgência sem o risco previsto de agravamento imediato; porém esses profissionais são capacitados e orientados por uma equipe multiprofissional. Comprova- se, a prevalência de condições clínicas nos atendimentos do SAMU. Entretanto, é importante ressaltar que as ocorrências oriundas dos acidentes de trânsito, quedas e transferências também apresentaram incidência elevada, fato que justifica a necessidade de investigação epidemiológica para o planejamento, capacitação e manutenção dessas modalidades de atendimentos. Ademais, é necessário parabenizar e incentivar a continuidade e a ampliação dos serviços desenvolvidos pelo SAMU, reconhecendo o grande valor do trabalho desses profissionais, juntamente a todos os outros envolvidos no APH do país.

\section{AGRADECIMENTOS E FINANCIAMENTO}

Faz- se menções de agradecimentos aos Coordenadores do Programa de Iniciação Científica do Centro Universitário UniEVANGÉLICA, Anápolis, Goiás, que possibilitou, estimulou e acompanhou o desenvolvimento desta pesquisa pelo Programa Voluntário de Iniciação Científica (PVIC), período 20192020, a Diretora do curso de enfermagem da UniEVANGÉLICA Dra Sandra Valéria Martins pela autorização desta, aos Coordenadores de campos de pesquisas da Secretaria Municipal de Saúde (SEMUSA) e especialmente a enfermeira Mirlene Garcia, que gentilmente facilitou o acesso dos pesquisadores aos dados arquivados no Núcleo de Vigilância Epidemiológica do Município. Muito obrigada a todos! 


\section{REFERÊNCIAS}

1. ALMEIDA PMV, et al. Análise dos atendimentos do SAMU 192: Componente móvel da rede de atenção às urgências e emergências. Esc. Anna Nery, 2016; 20(2): 289-295.

2. BATTISTI GR, et al. Perfil de atendimento e satisfação dos usuários do Serviço de Atendimento Móvel de Urgência (SAMU). Rev. Gaúcha Enferm., 2019; 40(1) e20180431): 1-8.

3. BRASIL. Portaria oㅡ 2048, de 5 de novembro de 2002. Ministério da Saúde. Secretaria de Atenção à Saúde.

4. BRASIL. Portaria ํo 1863, de 29 de setembro de 2003. Ministério da Saúde. Secretaria de Atenção à Saúde.

5. BRASIL. Programa mínimo para implantação das motolâncias na rede SAMU 192. s/d. Ministério da Saúde. Secretaria de atenção à saúde departamento de atenção especializada coordenação geral de urgência e emergência.

6. ESPERÓN JMT. Pesquisa quantitativa na ciência da enfermagem. Esc. Anna Nery, 2017; 21(1) e20170027: 1-2.

7. GOMES ATL, et al. Perfil epidemiológico das emergências traumáticas assistidas por um serviço préhospitalar móvel de urgência. Enfermería Global, 2017; 16(1): 395-405.

8. MALTA DC, et al. Mortalidade e anos de vida perdidos por violências interpessoais e autoprovocadas no Brasil e Estados: análise das estimativas do Estudo Carga Global de Doença, 1990 e 2015. Rev. bras. epidemiol., 2017; 20(1): 142-156.

9. O'DWYER G, et al. O processo de implantação do Serviço de Atendimento Móvel de Urgência no Brasil: estratégias de ação e dimensões estruturais. Cad. Saúde Pública, 2017; 33(7) e00043716: 1-14.

10. RIBEIRO AP, et al. Quedas acidentais nos atendimentos de urgência e emergência: resultados do VIVA Inquérito de 2014. Ciênc. saúde coletiva, 2016; 2 (12): 3719-3727.

11. SANTOS LFS, et al. Estudo epidemiológico do trauma ortopédico em um serviço público de emergência. Cad. saúde colet., 2016; 24 (4): 397-403.

12. TIENSOLI SD, et al. Características dos idosos atendidos em um pronto-socorro em decorrência de queda. Rev. Gaúcha Enferm., 2019; 40, e20180285: 8 p. 